\title{
Desempenho e características da carcaça e da carne de novilhos não-castrados alimentados com ou sem adição de monensina e/ou probiótico à dieta
}

\author{
Performance, carcass and meat traits of non-castrated cattle fed with or without monensin and/or \\ probiotic addition to the diet
}

\author{
Fernando Kuss ${ }^{\mathrm{I}}$ José Luis Moletta ${ }^{\mathrm{II}}$ Meiby Carneiro de Paula ${ }^{\mathrm{III}}$ Ivan César Furmann Moura ${ }^{\mathrm{IV}}$ \\ Stefano Juliano Tavares de Andradev ${ }^{\mathrm{V}}$ André Guimarães Maciel e Silvav
}

\section{RESUMO}

O objetivo do estudo foi avaliar o desempenho $e$ as características da carcaça e da carne de novilhos nãocastrados alimentados com ou sem adição de monensina (M) e/ou probiótico (P) (Sacharomyces cerevisiae) à dieta. Os animais foram distribuídos em baias individuais, permanecendo 145 dias em confinamento. A dieta foi composta de silagem de milho e 1,2\% do peso vivo de concentrado com base da matéria natural. Não houve efeito da adição dos aditivos fornecidos de forma isolada ou mesmo da associação destes sobre o consumo de alimento, o ganho de peso e a conversão alimentar. As médias de consumo, ganho de peso e conversão alimentar apresentaram comportamento quadrático com o avanço do período de confinamento. Embora a adição de $M$ ou $P$ à dieta tenha resultado no aumento numérico $(P>0,05)$ do consumo (4,2\%), a média de ganho de peso reduziu $(5,8$ e $5,3 \%$, respectivamente), resultando em pior conversão alimentar $(P>0,05)$. Já a associação de $M+P$ aumentou $(P>0,05)$ o consumo em $9,5 \%$, com concomitante aumento $(P>0,05)$ do ganho de peso $(6,4 \%)$ em relação à dieta controle. Os animais alimentados com $M+P$ apresentaram melhor acabamento de carcaça $(5,5 \mathrm{~mm})$, seguidos por aqueles do grupo controle $(4,7 \mathrm{~mm})$, sendo os valores inferiores verificados nas carcaças dos animais $M(3,7 \mathrm{~mm})$ e $P(3,5 \mathrm{~mm})$. A adição de monensina e/ou probiótico (Sacharomyces cerevisiae) na dieta de novilhos na fase de terminação em confinamento não proporciona melhora no desempenho e nas características da carcaça e da carne de novilhos.

Palavras-chave: bovinos de corte, confinamento, espessura de gordura, ionóforo, Sacharomyces cerevisiae.

\begin{abstract}
The objective of this experiment was to evaluate the performance, carcass and meat characteristics of noncastrated cattle with or without monensin (M) and/or probiotic (P) (Saccharomyces cerevisiae) to the diet. The animals were divided into individual stalls, being left 145 days in feedlot. The roughage used was corn silage $+1.2 \%$ of the weight live of concentrate in natural matter basis. There was no effect of the addition of the addictives supplied isolated or associated over feed intake, weight gain and fed conversion. Feed intake, weight gain and feed conversion averages presented quadratic behavior with the increase of the period feedlot. Although the addition of $M$ or $P$ to the diet resulted in the numeric increase $(P>.05)$ of the intake $(4.2 \%)$ the average of weight gain reduced (5.8 and $5.3 \%$, respectively), resulting in smaller fed conversion $(P>.05)$. The association of $M+P$ already increased $(P>.05)$ the food intake to the order of $9.5 \%$ with concomitant increase $(P>.05)$ of the weight gain $(6,4 \%)$ when compared to diet control. The animals fed with $M+P$ presented better carcass finish $(5.5 \mathrm{~mm})$, followed by the control $(4.7 \mathrm{~mm})$, and smaller values, it was verified in the carcasses of the animals that received $M(3.7 \mathrm{~mm})$ and $P(3.5 \mathrm{~mm})$. The addition of monensin and/or probiotic for feedlot steers did not improve the performance, carcass and meat quality.
\end{abstract}

Key words: beef cattle, fat thickness, feedlot, ionofore, Sacharomyces cerevisiae.

\section{INTRODUÇÃo}

A monensina é um ionóforo comumente utilizado na alimentação de ruminantes com o intuito

'Universidade Tecnológica Federal do Paraná (UTFPR), Campus Dois Vizinhos. Estrada para Boa Esperança, km 04, Comunidade de São Cristóvão, 85660-000, Dois Vizinhos, PR, Brasil. E-mail: fernandokuss@ig.com.br. Autor para correspondência.

"Instituto Agronômico do Paraná (IAPAR), Ponta Grossa, PR, Brasil.

"IIUniversidade Estadual de Londrina (UEL). Londrina, PR, Brasil.

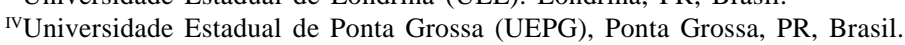

vFaculdade de Medicina Veterinária, Universidade Federal do Pará (UFPA), Castanhal, PA, Brasil. 
de melhorar a eficiência alimentar e aumentar o fluxo de aminoácidos dietéticos para o intestino delgado. Porém, a sua utilização na alimentação animal foi proibida nos Estados Unidos e nos países da União Européia a partir de 2006 ( artigo $^{\circ} 11$, regulamento 1831/2003) devido à restrição ao consumo por parte dos humanos de produtos de origem animal produzidos com uso de antibióticos como promotores de crescimento. Outro grupo de aditivo alimentar estudado a partir da década de 90 na alimentação de monogástricos e ruminantes são os probióticos. Estes normalmente são compostos por uma combinação de fungos e/ou bactérias e tem como objetivo promover um balanço da flora microbiana, proporcionando uma digestão mais eficiente dos nutrientes e assim melhorando a transformação de alimento consumido em produção de carne sem que esses microorganismos sejam absorvidos e acumulados no tecido (LÓPEZ, 2000).

Em trabalhos nacionais compilados por PASCOAL et al. (2000), os autores reportam incremento médio no ganho de peso de $4,5 \%$ e melhora na conversão alimentar de $7 \%$ ao longo da terminação em confinamento de novilhos e novilhas em confinamento. Na revisão realizada por GOODRICH et al. (1984), os autores demonstraram que o efeito da monensina sobre as características da carcaça é altamente variável e que os modelos de regressão utilizados para explicar o efeito da monensina indicaram que esse ionóforo reduz o rendimento de carcaça. $\mathrm{O}$ efeito de probióticos sobre as características de carcaça e carne ainda são escassos na literatura científica, e os resultados encontrados são contraditórios. No estudo de WOHLT et al. (1998), os autores demonstraram que a associação da monensina com Saccharomyces cerevisiae à dieta de vacas leiteiras aumentou o consumo de alimento, a digestibilidade da fibra em detergente ácido e reduziu a relação de ácido acético:propiônico no rúmen em relação à dieta controle, aumentando a densidade energética da dieta. Em revisão, KREHBIEL et al. (2003) reportam o efeito do uso de probióticos na alimentação sobre o peso de carcaça, mantendo-se o rendimento similar a dietas controle.

Este trabalho tem o objetivo de avaliar o desempenho e as características da carcaça e da carne de novilhos não-castrados terminados em confinamento, recebendo dietas com ou sem monensina e/ou probiótico (Sacharomyces cerevisiae).

\section{MATERIAL E MÉTODOS}

O estudo foi conduzido na Estação Experimental Fazenda Modelo do Instituto Agronômico do Paraná (IAPAR), em Ponta Grossa, Paraná (PR).
Foram utilizados 23 novilhos não-castrados, sendo oito $1 / 2$ Pardo Suíço + 1/2 Canchim, sete $1 / 2$ Purunã + 1/2 Canchim e oito $3 / 4$ Purunã $+1 / 4$ Canchim, com peso vivo médio de 265,1 kg, 22 meses de idade e 2,9 pontos de escore corporal. Os animais foram alocados em baias individuais, recebendo dietas sem ou com monensina sódica - M (200mg animal ${ }^{-1}$ dia $\left.^{-1}\right)$ e ou probiótico - P (Saccharomyces cerevisiae; 23,7 x $108 \mathrm{UFC}$ animal ${ }^{-1}$ dia $^{-1}$ ), via concentrado, formando os seguintes tratamentos: C (controle), M, P e M+P.

Os animais foram pesados no início do período experimental e em intervalos de 27, 28, 28, 33 e 29 dias, totalizando 145 dias de avaliação em confinamento. A cada pesagem os animais eram pesados após um jejum de sólidos de 16 horas para acompanhamento da evolução do peso vivo e do escore de condição corporal determinado por um avaliador treinado, seguindo a classificação proposta por LOWMAN et al. (1973). A dieta utilizada foi composta de silagem de milho ad libitum e 1,2\% do peso vivo de concentrado com base da matéria natural, sendo o seu ajuste de oferta realizado por ocasião das pesagens periódicas dos animais. Antecedendo a alimentação diária, as sobras de alimento foram retiradas, pesadas e registradas em planilha para cálculo do consumo de matéria seca dos animais.

Antecedendo o embarque para o frigorífico, os animais foram submetidos ao jejum de sólidos de 16 horas. Em seguida, eles foram pesados e transportados até o frigorífico comercial (duração de 30 minutos), procedendo-se o abate após descanso e à dieta hídrica de 12 horas. Ao fim da linha de abate, as duas meiascarcaças foram lavadas, identificadas e pesadas, sendo em seguida conduzidas à câmara fria por um período de 24 horas à temperatura de $2^{\circ} \mathrm{C}$. Após refrigeração, as duas meias-carcaças foram avaliadas quanto à conformação seguindo a metodologia descrita por MÜLLER (1987). Na carcaça direita, foi registrado a espessura de coxão, o comprimento da perna e carcaça interna. Em seguida, foi realizado um corte transversal ao contra-filé entre a $12^{\mathrm{a}}$ e a $13^{\mathrm{a}}$ costela, com o intuito de expor o músculo Longissumus dorsi para mensurar a espessura de gordura subcutânea, a área do Longissumus dorsi, o grau de marmorização, a textura e a coloração, segundo a metodologia de MÜLLER (1987).

O delineamento experimental utilizado foi o inteiramente casualizado. A análise estatística foi realizada utilizando o procedimento GLM do SAS (2000). As variáveis que não apresentaram distribuição normal foram analisadas pela metodologia de Modelos Lineares Generalizados, utilizando o procedimento GENMOD. Quando a ANOVA não identificou efeito 
dos tratamentos $(\mathrm{P}<0,10)$ sobre as variáveis dependentes relacionadas ao desempenho em confinamento, foram estimadas equações de regressão de todas as variáveis estudadas em função dos dias de terminação. Já para as variáveis dependentes relacionadas à carcaça e à carne, foi realizada a comparação entre as médias utilizando o procedimento LSMEANS do SAS (2000) em nível de significância de $5 \%$.

\section{RESULTADOS E DISCUSSÃO}

Observa-se na tabela 1 que, ao longo do período de confinamento, o consumo de matéria seca em relação ao peso vivo (CMSPV) e ao tamanho metabólico (CMSTM) se comportou de forma quadrática. Nos primeiros 27 dias de confinamento, se observou o maior consumo, seguido de queda dos 27 aos 55 dias, mantendo-se estável até os 116 dias e reduzindo novamente dos 116 aos 145 dias de terminação. $\mathrm{O}$ aumento de consumo no primeiro período foi esperado, uma vez que antecedendo o início do experimento os animais foram mantidos em pastagem nativa, ocorrendo, dessa forma, restrição qualitativa de nutrientes, sendo estes forçados a consumir mais matéria seca para suprir suas exigências, resultando em maior desenvolvimento e capacidade do trato gastrintestinal (ganho compensatório). Após o efeito do processo compensatório (primeiro período), ambos os CMSPV e CMSTM foram reduzidos e mantiveramse estáveis até os 116 dias, indicando recuperação dos tecidos corporais, principalmente os órgãos vitais e o trato gastrintestinal, diminuindo o espaço abdominal e conseqüentemente o consumo. No último período (116 aos 145 dias), o CMSPV e o CMSTM sofreram nova redução, porém nessa fase a deposição de gordura abdominal passou a ser o limitante do consumo de alimento, causando a redução do apetite ou o chamado feedback do tecido adiposo (NRC, 1996).

$\mathrm{O}$ peso dos animais, independente do tratamento, foi crescente ao longo dos dias de terminação, resultado do similar ganho de peso (GMD) entre os tratamentos (Tabela 2). Mesmo comportamento se verificou para o escore de condição corporal (ECC), em que a média de ECC ao abate foi de 4,4 pontos (considerados gordos). O aumento do ECC ao decorrer da terminação é esperado em razão da mudança da composição corporal dos animais, aumentando a deposição de tecido adiposo com o aumento do peso.

Tabela 1 - Consumo diário de matéria seca expresso em valor absoluto (CMS), CMS em relação ao peso vivo (CMSPV) e CMS em relação ao tamanho metabólico (CMSTM), de acordo com os tratamentos e dias de confinamento (D).

\begin{tabular}{|c|c|c|c|c|c|c|}
\hline \multirow{2}{*}{ Tratamento } & \multicolumn{5}{|c|}{ 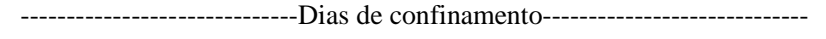 } & \multirow{2}{*}{ Médias } \\
\hline & 27 & 55 & 83 & 116 & 145 & \\
\hline & \multicolumn{6}{|c|}{ 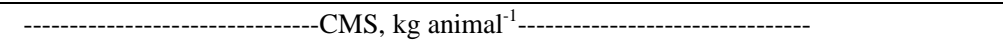 } \\
\hline Controle & 8,19 & 7,14 & 7,41 & 8,29 & 7,40 & 7,69 \\
\hline Monensina (M) & 8,72 & 7,92 & 7,14 & 8,27 & 7,60 & 7,93 \\
\hline Probiótico (P) & 8,51 & 7,14 & 7,24 & 8,45 & 8,22 & 7,91 \\
\hline $\mathrm{M}+\mathrm{P}$ & 9,00 & 7,80 & 7,63 & 9,09 & 9,21 & 8,55 \\
\hline Médias & 8,63 & 7,53 & 7,36 & 8,54 & 8,13 & \\
\hline Equação de regressão & \multicolumn{5}{|c|}{$\hat{Y}=9,2848-0,0385 D^{*}+0,0002 D^{2}$} & \\
\hline & & & MSPV & 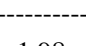 & ----- & \\
\hline Controle & 2,84 & 2,11 & 1,95 & 1,98 & 1,74 & 2,13 \\
\hline Monensina (M) & 3,00 & 2,39 & 1,94 & 2,01 & 1,75 & 2,22 \\
\hline Probiótico (P) & 2,96 & 2,16 & 1,98 & 2,08 & 1,91 & 2,22 \\
\hline$M+P$ & 3,08 & 2,32 & 2,02 & 2,14 & 2,10 & 2,33 \\
\hline Médias & 2,98 & 2,26 & 1,97 & 2,05 & 1,89 & \\
\hline Equação de regressão & \multicolumn{5}{|c|}{$\hat{Y}=3,6197-0,0297 \mathrm{D}+0,0001 \mathrm{D}^{2}$} & \\
\hline Controle & 117,08 & 90,56 & 86,14 & 89,39 & 73,81 & 91,40 \\
\hline Monensina (M) & 123,97 & 101,88 & 84,88 & 90,38 & 76,60 & 95,54 \\
\hline Probiótico (P) & 121,72 & 92,17 & 86,57 & 93,21 & 83,12 & 95,36 \\
\hline$M+P$ & 127,36 & 99,27 & 89,07 & 96,95 & 89,21 & 100,37 \\
\hline Médias & 122,36 & 96,39 & 86,69 & 92,59 & 80,89 & \\
\hline Equação de regressão & & $\hat{Y}=$ & 0,9605 & $39 D^{2}$ & & \\
\hline
\end{tabular}

Ciência Rural, v.39, n.4, jul, 2009. 
Tabela 2 - Evolução do peso, escore de condição corporal (ECC), ganho de peso diário (GMD) e conversão alimentar (CA) de acordo com os tratamentos e dias de confinamento (D).

\begin{tabular}{|c|c|c|c|c|c|c|c|}
\hline \multirow{3}{*}{ Tratamento } & & \multicolumn{5}{|c|}{ 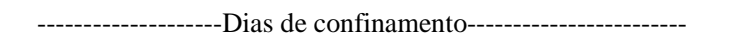 } & \multirow{3}{*}{ Médias } \\
\hline & & \multirow[b]{2}{*}{27} & \multirow[b]{2}{*}{55} & \multirow[b]{2}{*}{83} & \multirow[b]{2}{*}{116} & \multirow[b]{2}{*}{145} & \\
\hline & & & & & & & \\
\hline & PESO inicial, kg & --- & ------- & ESO, $\mathrm{k}$ & 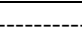 & - & \\
\hline Controle & 264,4 & 311,4 & 359,6 & 395,2 & 439,2 & 489,4 & -- \\
\hline Monensina (M) & 266,2 & 312,7 & 350,8 & 387,0 & 437,3 & 481,0 & -- \\
\hline Probiótico (P) & 261,8 & 306,8 & 347,0 & 377,8 & 431,2 & 476,0 & -- \\
\hline$M+P$ & 267,3 & 312,7 & 356,2 & 395,7 & 451,5 & 510,3 & -- \\
\hline Médias & 265,1 & 311,0 & 353,4 & 389,1 & 440,2 & 489,8 & \\
\hline \multirow[t]{2}{*}{ Equação de regressão } & \multicolumn{6}{|c|}{$\hat{Y}=267,1167+1,5164 \mathrm{D}$} & \\
\hline & ECC inicial, pontos & --- & ------- & C, pon & ------ & -- & \\
\hline Controle & 2,8 & 3,2 & 3,5 & 3,8 & 4,1 & 4,4 & -- \\
\hline Monensina (M) & 2,9 & 3,2 & 3,5 & 3,8 & 4,3 & 4,4 & -- \\
\hline Probiótico (P) & 3,0 & 3,1 & 3,5 & 3,7 & 4,1 & 4,4 & -- \\
\hline$M+P$ & 2,9 & 3,1 & 3,5 & 3,7 & 4,2 & 4,5 & -- \\
\hline Médias & 2,9 & 3,1 & 3,5 & 3,7 & 4,2 & 4,4 & -- \\
\hline Equação de regressão & & & \multicolumn{4}{|c|}{$\hat{Y}=2,8781=0,0109 \mathrm{D}$} & \\
\hline Controle & -- & 1,74 & 1,72 & 1,27 & 1,33 & 1,73 & 1,56 \\
\hline Monensina (M) & -- & 1,72 & 1,36 & 1,29 & 1,52 & 1,51 & 1,48 \\
\hline Probiótico (P) & -- & 1,67 & 1,44 & 1,10 & 1,62 & 1,54 & 1,47 \\
\hline$M+P$ & -- & 1,68 & 1,55 & 1,41 & 1,69 & 2,02 & 1,67 \\
\hline Médias & -- & 1,70 & 1,51 & 1,28 & 1,55 & 1,71 & \\
\hline \multirow[t]{2}{*}{ Equação de regressão } & & \multicolumn{5}{|c|}{$\hat{Y}=2,09883-0,01712 \mathrm{D}+0,00010075 \mathrm{D}^{2}$} & \\
\hline & & & & --CA-- & ------ & --- & \\
\hline Controle & -- & 4,76 & 4,26 & 6,22 & 6,24 & 4,34 & 5,16 \\
\hline Monensina (M) & -- & 5,08 & 6,23 & 5,65 & 5,68 & 5,53 & 5,63 \\
\hline Probiótico (P) & -- & 5,27 & 5,16 & 6,80 & 5,20 & 5,44 & 5,57 \\
\hline$M+P$ & -- & 5,37 & 5,20 & 5,88 & 5,45 & 4,53 & 5,22 \\
\hline Médias & -- & 5,13 & 5,26 & 6,02 & 5,64 & 4,97 & \\
\hline Equação de regressão & & & $\hat{Y}=4$ & 0,039 & $39 D^{2}$ & & \\
\hline
\end{tabular}

O ganho de peso diário (GMD) dos animais apresentou comportamento quadrático ao longo dos dias de terminação. A redução do GMD dos 27 aos 55 dias de terminação era esperada em razão do término do processo de ganho compensatório, indicando a recuperação dos tecidos vitais e, consequentemente, aumentando a exigência de mantença dos animais, reduzindo assim a oferta de energia para ganho. Já a queda do GMD dos 55 aos 83 dias é explicada pela alteração da composição da fração concentrada. Nesse período, o farelo de soja foi substituído por grão de soja triturado, aumentando dessa forma a quantidade de óleo na dieta, o que possivelmente tenha provocado alteração do ambiente ruminal e redução da digestibilidade de nutrientes (VAN SOEST, 1994). A partir dos 83 dias a composição do concentrado voltou a contar com o farelo de soja, o que promoveu o incremento do GMD nos períodos subseqüentes, resultado do aumento concomitante do consumo (Tabela 1). Todavia, o GMD médio ao longo da terminação neste estudo foi de $1,55 \mathrm{~kg}$, considerado satisfatório levando em consideração a relação volumoso:concentrado utilizada (52:48). QUEIROZ et al. (2004) também não encontraram efeito da adição de enzimas + leveduras na dieta sobre o desempenho de novilhos em confinamento, demonstrando comportamento quadrático segundo a equação de regressão com o avanço dos dias de terminação. Porém, os autores ressaltam que o GMD dos animais suplementados com enzimas + levedura apresentou superioridade de 5,2\% em relação à dieta controle, ao passo que no presente estudo verificou-se redução de 5,8\% em comparação com a dieta controle. A dieta com monensina também apresentou tendência $(\mathrm{P}>0,05)$ de 
redução de GMD $(5,1 \%)$ em relação à dieta controle. Já RESTLE et al. (2001) verificaram redução significativa e mais expressiva (10,3\%) quando adicionada monensina na dieta de vacas de descarte terminadas em confinamento. No entanto, quando houve a associação de monensina $(\mathrm{M})$ + probiótico $(\mathrm{P})$ à dieta, o incremento no GMD foi da ordem de 6,4\% em relação aos animais alimentados com $\mathrm{M}$ ou $\mathrm{P}$, possivelmente isso seja resultado dos efeitos da monensina (regulação do $\mathrm{pH}$ ruminal) e do probiótico (digestibilidade dos nutrientes) que ocasionaram o aumento de 8\% no CMS (Tabela 1). A conversão alimentar (CA) acompanhou o mesmo comportamento do GMD e CMS ao longo da terminação dos animais, o que se deve ao fato de a CA ser a relação entre estas duas variáveis. A CA média durante o confinamento dos animais foi excelente $(5,4)$. CA semelhante $(5,2)$ ao presente estudo foi observado por PACHECO et al. (2005) com novilhos da mesma categoria terminados em confinamento, porém com relação volumoso:concentrado ainda inferior (60:40). Segundo os autores, essa melhor eficiência foi proporcionada pelo ganho compensatório dos animais.

Observa-se na tabela 3 que não houve diferença significativa para peso ao abate e peso de carcaça quente, indicando não haver benefício da adição de monensina (M) e ou ${ }^{-1}$ probiótico (P) na dieta sobre essas variáveis. Similar comportamento foi observado por BEAUCHEMIN et al. (1997) e ELAM et al. (2003) para essas variáveis quando adicionaram enzimas fibrolíticas e leveduras, respectivamente, à dieta de novilhos. Trabalhando com vacas de descarte alimentadas em confinamento sem ou com monensina (200mg animal ${ }^{-1}$ dia $^{-1}$ ), KUSS et al. (2009) observaram redução significativa no peso de abate e carcaça de 30,0 e $27,5 \mathrm{~kg}$, respectivamente, para os animais alimentados com monensina. Porém, KREHBIEL et al. (2003), analisando vários estudos com a adição de probióticos, constataram efeito positivo significativo desse aditivo sobre essas características. Ainda na tabela 3 verifica-se que a espessura de gordura subcutânea (EGS) foi maior para os tratamentos M+P e controle (C), e a espessura inferior foi constatada nas carcaças dos animais proveniente dos tratamentos $\mathrm{M}$ e P. Considerando-se que, no processo de terminação, parte do ganho de peso corresponde a gordura, tendo os animais dos tratamentos $\mathrm{M}+\mathrm{P}$ e $\mathrm{C}$ maiores taxas de ganho de peso, espera-se que a deposição de gordura de cobertura desses grupos seja mais precoce em relação à dos animais dos tratamentos $\mathrm{M}$ e $\mathrm{P}$. As carcaças de todos os tratamentos se mantiveram dentro da faixa exigida pelos frigoríficos (3 a $6 \mathrm{~mm}$ ). Os resultado observados divergem dos descritos por ELAM et al. (2003), os quais demonstraram que a adição de leveduras resulta em melhor acabamento da carcaça de novilhos terminados em confinamento. O rendimento de carcaça foi similar entre os tratamentos avaliados neste estudo. Nos estudos realizados por KREHBIEL et al. (2003) e ELAM et al. (2003), também não foi observado alteração do rendimento de carcaça com a adição de probiótico à dieta de novilhos. Quanto à utilização de monensina, KUSS et al. (2009) descreveram redução significativa do rendimento de carcaça com a adição de monensina à dieta de vacas. A conformação não sofreu efeito de tratamento, demonstrando valor médio excelente de 14,0 pontos (muito boa típica). Resultado este, reflexo dos grupos genéticos utilizados e equilibrados nos tratamentos, sendo todos de aptidão para alto desenvolvimento muscular na carcaça.

Na tabela 4 são apresentados os resultados referentes às medidas métricas da carcaça e qualitativas da carne. Observa-se que os valores foram semelhantes entre os tratamentos para comprimento de carcaça e perna, espessura de coxão e área de Longissimus dorsi. Quanto às características qualitativas, estas não sofreram efeito de tratamento. A associação da espessura de gordura com a coloração da carne foi positiva ( $\mathrm{r}=0,48 ; \mathrm{P}=0,0222)$, demonstrando que carcaças

Tabela 3 - Médias para características quantitativas da carcaça de novilhos alimentados com ou sem monensina e/ou probiótico.

\begin{tabular}{|c|c|c|c|c|}
\hline \multirow{2}{*}{ Variáveis } & \multirow[b]{2}{*}{ Controle } & \multirow[b]{2}{*}{ Monensina (M) } & \multirow[b]{2}{*}{ Probiótico (P) } & \multirow[b]{2}{*}{$\mathrm{M}+\mathrm{P}$} \\
\hline & & & & \\
\hline Peso de abate, kg & 490,3 & 479,6 & 480,2 & 507,5 \\
\hline Peso de carcaça quente, $\mathrm{kg}$ & 267,3 & 263,9 & 263,8 & 273,0 \\
\hline Espessura de gordura (EGS), mm & $4,7 \mathrm{ab}$ & $3,7 b$ & $3,5 b$ & $5,5 \mathrm{a}$ \\
\hline EGS $100 \mathrm{~kg}^{-1}$ de carcaça, mm & $1,8 \mathrm{ab}$ & $1,41 b$ & $1,34 \mathrm{~b}$ & $2,0 \mathrm{a}$ \\
\hline Rendimento de carcaça quente, \% & 54,6 & 55,0 & 54,8 & 53,9 \\
\hline Conformação, pontos2 & 14,0 & 13,7 & 14,0 & 14,3 \\
\hline
\end{tabular}

1Médias seguidas por letras minúsculas diferem entre si $(\mathrm{P}<0,05) .213=$ muito boa - ; 15 = muito boa + .

Ciência Rural, v.39, n.4, jul, 2009. 
Tabela 4 - Médias para as características métricas e qualitativas da carcaça de novilhos alimentados com ou sem monensina e/ou probiótico.

\begin{tabular}{|c|c|c|c|c|}
\hline \multirow{2}{*}{ Variáveis } & \multirow[b]{2}{*}{ Controle } & \multirow[b]{2}{*}{ Monensina (M) } & \multirow[b]{2}{*}{ Probiótico (P) } & \multirow[b]{2}{*}{$\mathrm{M}+\mathrm{P}$} \\
\hline & & & & \\
\hline Comprimento de carcaça, cm & 134,31 & 134,0 & 131,6 & 135,7 \\
\hline Comprimento de perna, cm & 66,2 & 65,3 & 65,4 & 60,2 \\
\hline Espessura de coxão, cm & 26,1 & 26,4 & 26,7 & 26,6 \\
\hline Área do Longissimus dorsi, $\mathrm{cm}^{2}$ & 75,0 & 77,6 & 75,5 & 76,0 \\
\hline Coloração, pontos2 & 3,8 & 3,4 & 3,1 & 3,7 \\
\hline Textura, pontos3 & 4,2 & 4,5 & 4,2 & 4,2 \\
\hline Marmoreio, pontos4 & 6,2 & 6,6 & 5,4 & 7,2 \\
\hline
\end{tabular}

1 Médias seguidas por letras minúsculas diferem entre si $(\mathrm{P}<0,05) .21$ =escura, ..., 5=vermelho vivo. $31=$ muito grosseira, ..., $5=$ =muito fina. 4 1 a $3=$ traços, ..., 16 a $18=$ abundante.

com melhor acabamento apresentam carne de melhor coloração. Segundo LAWRIE (2005), carcaças com melhor acabamento são menos propensas à formação da metamioglobina (que promove o aspecto escuro da carne) devido à menor oxigenação dos músculos externos que recobrem a carcaça com o meio externo da câmara fria. A textura da carne se mostrou fina, resultado esperado, uma vez que os animais foram abatidos com idade jovem (27 meses). A deposição de gordura de marmorização variou de leve a pequena, quantidade bem aceita pela maioria dos consumidores. BEAUCHEMIN et al. (1997) eELAM et al. (2003) também não relatam efeito do uso de enzimas e leveduras, respectivamente, sobre o grau de marmorização da carne de novilhos terminados em confinamento.

\section{CONCLUSÕES}

A adição de monensina e/ou probiótico (Saccharomyces cerevisiae) na alimentação de novilhos não-castrados, na fase de terminação em confinamento, não proporciona melhora no desempenho em confinamento, bem como não possui efeito sobre a qualidade da carcaça e da carne. Sugerese que sejam realizados estudos com níveis maiores de concentração de Saccharomyces cerevisiae na dieta, uma vez que os resultados na literatura ainda são contraditórios.

\section{REFERÊNCIAS}

BEAUCHEMIN, K.A. et al. Effects of fibrolytic enzymes in corn or barley diets on performance and carcass characteristics of feedlot cattle. Canadian Journal of Animal Science, v.77, p.645-653, 1997.

ELAM, N.A. et al. Effects of live cultures of Lactobacillus acidophilus (strains NP45 and NP51) and Propionibucterium freudenreichii on perfomnce, carcass, and intestinal characteristics, and Escherichia coli strain 0157 shedding of finishing beef steers. Journal of Animal Science. v.81, p.2686-2698, 2003. Disponível em: < http://jas.fass.org/cgi/ reprint $/ 81 / 11 / 2686$ ? maxtoshow $=\&$ HITS $=10 \&$ hits $=10 \& R$ E S U L T F O R M A T = \& a u t h o r $1=$ E L A M $\% 2 \mathrm{C}+$ N.A.\&searchid $=1 \&$ FIRSTINDEX $=0$ \&resourcetype $=$ HWCIT $>$ Acesso em: 28 nov. 2008.

GOODRICH, R.D. et al. Influence of monensina the performance of cattle. Journal of Animal Science. v.58, n.6, p.1484-1498, 1984. Disponível em: <http://jas.fass.org/ $\mathrm{cgi} /$ reprint $/ 58 / 6 / 1484$ ? maxtoshow $=\&$ HITS $=10 \&$ hits $=10 \& \mathrm{R}$ E S U L T F O R M A T $=\&$ a u t h or $1=$ G O O D R I C H \%2C+R.D.+\&searchid=1\&FIRSTINDEX=0\&resourcetype=HWCIT $>$. Acesso em: 28 nov. 2008.

KREHBIEL, C.R. et al. Bacterial direct-fed microbials in ruminant diet: performance response and mode of action. Journal of Animal Science. v.81, E.Supl.2, E120-E132, 2003. Disponível em: <http://jas.fass.org/cgi/reprint/81/ 14_suppl_2/E120? maxtoshow $=\&$ HITS $=10$ \&hits $=10 \& R$ E S U L T F O R M A T $=\&$ a u th or $1=$ K R E H B I E L \% $2 \mathrm{C}+$ C.R. + \&searchid $=1 \&$ FIRSTINDEX $=0$ \&resourcetype $=$ HWCIT $>$. Acesso em: 28 nov. 2008.

KUSS, F. et al. Características da carcaça de vacas de descarte terminadas em confinamento recebendo dietas com ou sem adição de monensina. Ciência Animal Brasileira, (NO PRELO), 2009.

LAWRIE, R.A. Ciência da carne. 6.ed. São Paulo: Artmed, 2005. 384p.

LÓPEZ, J. Probiotics in animal nutrition. Asian Australasian Journal Animal Science, v.13, p.12-26, 2000.

LOWMAN, B.G. et al. Condition scoring beef cattle. Edinburgh: East of Scotland Colleg of Agriculture, 1973. 8p.

MÜLLER, L. Normas para a avaliação de carcaças e concurso de carcaças de novilhos. 2.ed. Santa Maria: UFSM, 1987. 31p.

NRC - NATIONAL RESEARCH COUNCIL. Nutrient requeriments of beef cattle. 7.rev ed. Washington, D.C: National Academy, 1996. 242p. 
PACHECO, P.S. et al. Desempenho de novilhos jovens e superjovens de diferentes grupos genéticos terminados em confinamento. Revista Brasileira de Zootecnia, v.34, n.3, p.963-975, 2005. Disponível em: < http://www.scielo.br/ scielo.php? s cript = sci_art text \& pid = S 1516 35982005000300030\&lng=pt\&nrm=iso>. Doi: 101590/ S1516-35982005000300030.

PASCOAL, L.L. et al. Uso de ionóforos e minerais no confinamento e em pastagem cultivada. In.: RESTLE, J. Eficiência na produção de bovinos de corte. Santa Maria: UFSM, 2000. p.304-332.

QUEIROZ, R.C. et al. Uso de produto à base de enzima e levedura na dieta de bovinos: digestibilidade dos nutrientes e desempenho em confinamento. Revista Brasileira de Zootecnia, v.33, n.4, p.1548-1556, 2004. Disponível em: $<\mathrm{h} \mathrm{t}$ t p : / / w w w. s c i e l o . b r / scielo.php? script =sci_arttext\&pid=S 1516 35982004000600022\&lng=pt\&nrm=iso>. Doi: 101590/ S1516-35982004000600022.

RESTLE, J. et al. Terminação em confinamento de vacas e novilhas sob dietas com ou sem monensina sódica. Revista Brasileira de Zootecnia, v.30, n.6, p.1801-1812, 2001.
Disponível em: <http://www.scielo.br/ scielo.ph p ? script = s ci_arttext\&pid=S 1516 35982001000700018\&lng=pt\&nrm=iso>. Doi: 101590/ S1516-35982001000700018.

SAS INSTITUTE. SAS/STAT. User's guide: statistics, versão 8.1. 4.ed. Cary: SAS Institute, 2000. V.2.

WOHLT, J.E. et al. Effect of yeast on feed intake and performance of cows fed diets based on corn silage during early lactation. Journal Dairy Science, v.81, p.1345-1352, 1998. Disponível em: < http://jds.fass.org/cgi/reprint/81/5/ 1345 ? maxtoshow $=\&$ HITS $=10 \&$ hits $=10 \&$ RESULTFO $\mathrm{RMAT}=$ \&author1=WOHLT\%2C $+\mathrm{J} . \mathrm{E} .+$ \&andorexacttitle $=\mathrm{a}$ nd \&andorexacttitleabs $=$ and $\&$ andorexactfulltext $=$ and \&searchid=1\&FIRSTINDEX=0\&sortspec=relevance $\&$ fdate $=1$ / $1 / 1998 \&$ tdate $=12 / 31 / 1998 \&$ resourcetype $=$ HWCIT $>$. Acesso: 28 nov. 2008.

VAN SOEST, P.J. Nutritional ecology of the ruminant. New York: Cornell University, 1994. 476p. 